\title{
Use of daptomycin and vancomycin in blood stream infections with PDR Acinetobacter Baumanii
}

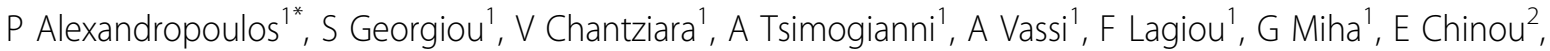 \\ K Skotadi², G Michaloudis ${ }^{1}$
}

From ESICM LIVES 2015

Berlin, Germany. 3-7 October 2015

\section{Introduction}

Multidrug-resistant and lately pan drug-resistant (PDR) Acinetobacter Baumanii presents an increasing challenge to health care and especially in Intensive Care Units (ICU).

\section{Objectives}

To evaluate the efficacy of daptomycin and vancomycin against PDR Acinetobacter Baumanii blood stream infections in ICU. In vitro, the administration of antibiotics usually used against Gram positive cocci (like vancomycin or daptomycin) has resulted in good bacterial response against PDR Acinetobacter even though it's a Gram - bacterium. However, clinical data to support the use of these regimens are still lacking.

\section{Methods}

From June 2014 to March 2015 all PDR Acinetobacter Baumanii blood stream infections along with the given therapeutic regimens were recorded. Age, sex, total days of antibiotic administration and culture results were recorded.

\section{Results}

4 blood stream infections in 4 patients (all women aged 34-78 average 57.75) were recorded for the time interval mentioned. Among those, 3 were originally receiving intravenous colistin plus meropenem while one was only taking meropenem. As soon as PDR blood stream infection was confirmed daptomycin $6 \mathrm{mg} / \mathrm{kg}$ (average 9 days of administration) was started on 2 patients while vancomycin $15 \mathrm{mg} / \mathrm{kg} / 12$ hours (average 8 days of administration)was added to the other two cases. Subsequent blood cultures came back negative after 2-3 days (2.2 days) of treatment.

\section{Conclusions}

Despite the small number of patients daptomycin and vancomycin seems to be effective against PDR Acinetobacter Baumanii blood stream infections. Further studies are needed to support the proposed scheme.

\section{Authors' details \\ ${ }^{1}$ Saint Savvas Hospital, Intensive Care Unit, Athens, Greece. ${ }^{2}$ Saint Savvas Hospital, Microbiology Dept, Athens, Greece.}

Published: 1 October 2015

\section{Reference}

1. Yang $H$, Chen $G, H u L$, Liu Y, Cheng J, Li H, et al: In vivo activity of daptomycin/colistin combination therapy in a Galleria mellonella model of Acinetobacter baumannii infection. Int I Antimicrob Agents 2015, 45(2):188-191.

doi:10.1186/2197-425X-3-S1-A407

Cite this article as: Alexandropoulos et al:: Use of daptomycin and vancomycin in blood stream infections with PDR Acinetobacter Baumanii. Intensive Care Medicine Experimental 2015 3(Suppl 1):A407. 